Forum 2018 · 33:313

https://doi.org/10.1007/s12312-018-0479-z

Online publiziert: 24. August 2018

(c) Springer Medizin Verlag GmbH, ein Teil von Springer Nature 2018

\title{
„Qualität heißt: aus der Versorgung lernen"
}

\section{Abstracteinreichung für den 4. interdisziplinären Kongress Quality of Cancer Care 2019 ist gestartet}

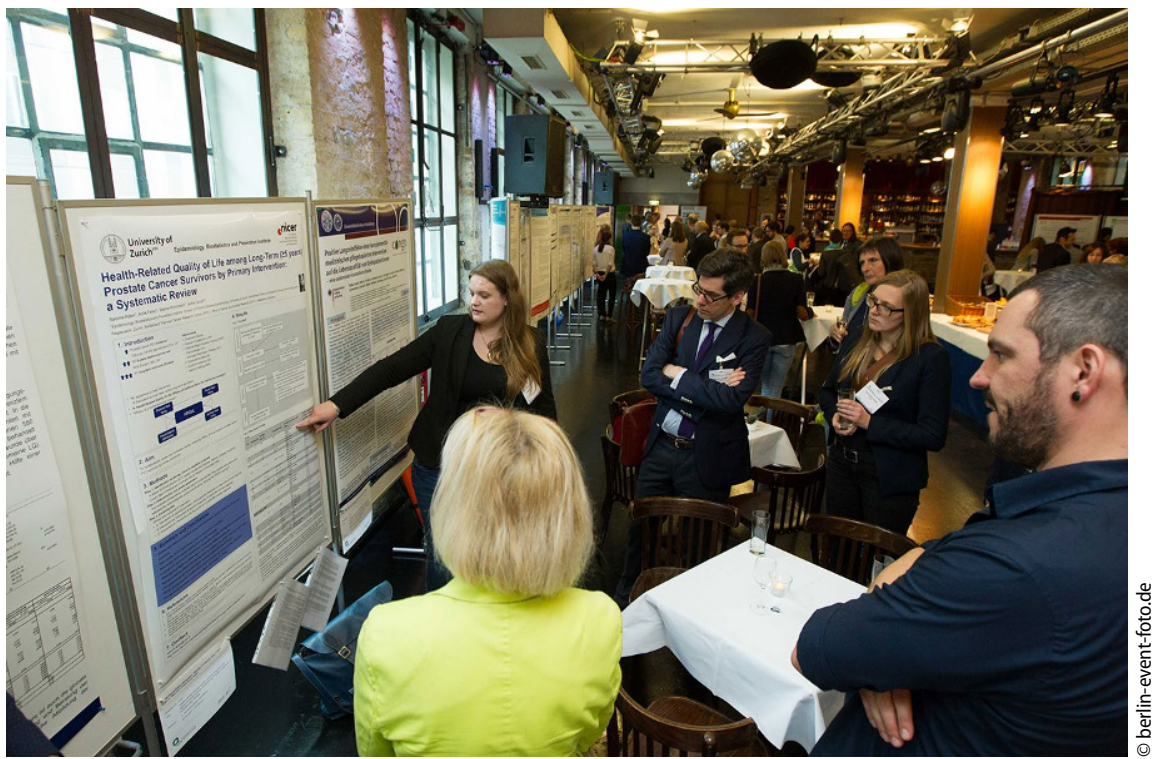

Krebsversorgung zu Reibungsverlusten kommt. Das gewählte Format des QoCC, eine Kombination aus Tutorials, Vorträgen, Diskussionsrunden und Posterpräsentationen, bietet eine ausgezeichnete Plattform für einen konstruktiven Gedanken- und Erfahrungsaustausch zur Minimierung von Hindernissen und Reibungsverlusten.

\section{Korrespondenzadresse}

Renate Babnik

Deutsche Krebsgesellschaft e.V. babnik@krebsgesellschaft.de

\begin{abstract}
Abb. 1 ॥ Geführte Posterbegehung beim QoCC 2017
forschung, Epidemiologen, Biometriker, Gesundheitsökonomen, Kliniker und Gesundheitspolitiker.

Ausrichter des QoCC sind die Deutsche Krebsgesellschaft e. V. und die Arbeitsgemeinschaft Deutscher Tumorzentren e. V. Anders als andere Veranstaltungen greift der QoCC keine einzelnen Aspekte auf, vielmehr geht es um eine Verknüpfung von onkologischen Leitlinien, Zertifizierung und Dokumentation, klinischen Studien und Versorgungsforschung. Die Idee zu diesem Kongress entstand aus der Erfahrung, dass es häufig die Schnittstellen zwischen den Bereichen sind, an denen es in der Qualitätsentwicklung der

Zentren, Clinical-Research-Organisationen, Einrichtungen der Versorgungsheißt: aus der Versorgung lernen“. QoCCTeilnehmer sind Experten von Krebsregistern, Tumorzentren, zertifizierten
\end{abstract}

